between asthmatic and non-asthmatic cells, and a biologically relevant difference in methylation of greater that $10 \%$ ( $\beta$ value $>0.1$ ). 10 of these sites were selected as top hits. 7 sites positively validated by pyrosequencing. They were associated with 7 different genes; LGALS3BP, ATP11A, ZNF696, KLF6, TBX1, RUNX3, and SPINT2. Expression of these genes was measured in ASM cells isolated from asthmatic and non-asthmatic donors. LGALS3BP expression was undetectable while ATP11A and ZNF696 displayed no difference in expression between cells from asthmatic and non-asthmatic donors. KLF6 and SPINT2 showed a trend towards increased expression in cells from asthmatic donors while RUNX3 and TBX1 showed a trend towards decreased expression.

Conclusions Differences in CpG methylation exist between ASM isolated from asthmatic and non-asthmatic donors. Future work will focus on identifying differentially methylated regions of DNA and further defining the association to gene and protein expression.
EFFECTS OF TIOTROPIUM ON ASTHMA EXACERBATIONS ARE NOT EXPLAINED BY AIRWAY HYPERRESPONSIVENESS, EXHALED BREATH NITRIC OXIDE OR AIRWAY GEOMETRY

S Jabbal, A Manoharan, BJ Lipworth. Scottish Centre for Respiratory Research, Dundee, UK

10.1136/thoraxjnl-2016-209333.382

Background Long acting muscarinic antagonists (LAMA) such as tiotropium (TIO) reduce asthma exacerbations in patients receiving inhaled corticosteroids and long-acting beta-agonists (ICS/ LABA). However the mechanism for this protective action of LAMA remains unclear.

Objectives To evaluate the response to indacaterol (IND) either alone in combination with tiotropium (IND/TIO) in addition to ICS on airway hyperresponsiveness (AHR), FeNO and impulse oscillometry (IOS).

\title{
Mannitol Sensitivity
}

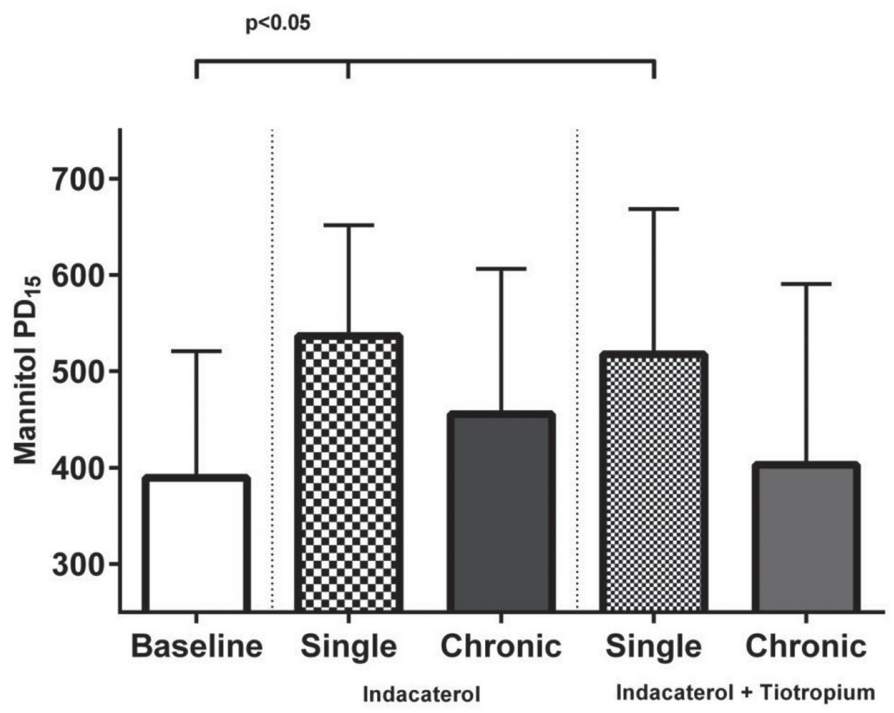

Mannitol Reactivity

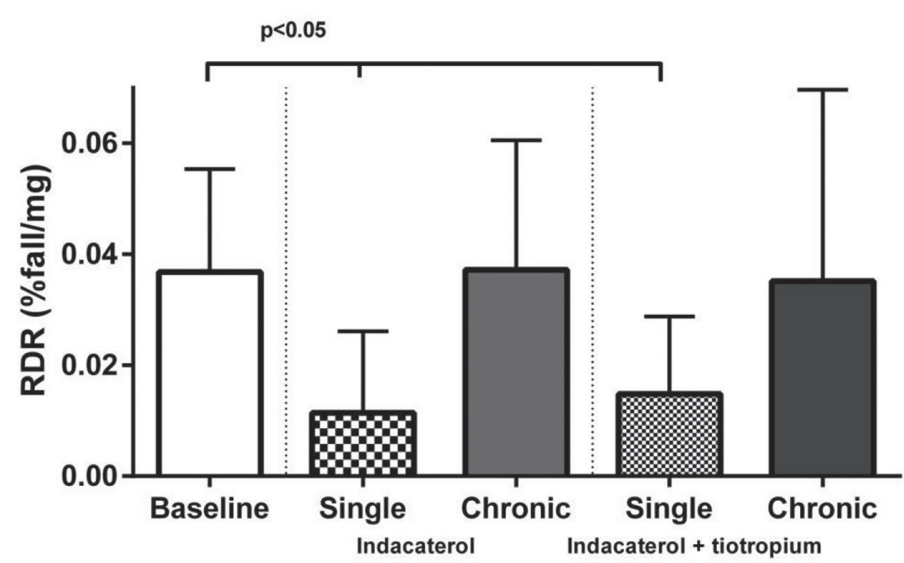

Abstract P239 Figure 1 Effects of randamised treatment compared to baseline on mannitol sensitivity and reactivuity. Values presented as geometric mean and $95 \%$ confidence interval. P value denotes significant difference for randamised treatment compatred to baseline. There was also a significant difference between single and chronic dosing for RDR with both treatments. 
Methods $\mathrm{n}=14$ ICS treated asthmatic patients (Mean age 46 years, FEV1 86\% predicted, R5 160\% predicted, ICS 693ug/ day), were randomised in cross-over fashion to receive either IND 150 ug alone (ICS/LABA) or in combination with TIO 18 ug once daily (ICS/LABA/LAMA) for 4 weeks with 2 week run-in and washout periods. Mannitol sensitivity (PD15) and reactivity (RDR), airway resistance (R5,R5-R20), reactance (AXE) and FeNO were measured at 24 hours after the first and last doses.

Results There were significant improvements in mannitol PD15 and RDR with IND or IND/TIO vs baseline after single but not chronic dosing (Figure). There was also a significant difference in RDR between single and chronic dosing for both treatments. R5,R5-R20 and AXE were significantly improved with both treatments compared to baseline after single and chronic dosing . There were no significant differences between treatments after chronic dosing for either mannitol or IOS . In contrast FeNO was unchanged with either treatment compared to baseline.

Conclusions There were significant improvements in mannitol sensitivity and reactivity with either IND or IND/TIO after single but not chronic dosing, while FeNO remained unchanged . Airway resistance and reactance were significantly decreased to the same degree with both treatments after chronic dosing. This in turn suggests that the mechanism by which LAMA reduces exacerbations is unlikely to be related to AHR, FeNO or airway geometry.

\section{P240 \\ LOW IGE AND NOT BLOOD EOSINOPHILS PREDICTS LACK OF RESPONSE TO OMALIZUMAB IN UHSM COHORT}

${ }^{1}$ TB Morris, ${ }^{1} \mathrm{HV}$ Patel, ${ }^{1} \mathrm{M}$ Demirbag, ${ }^{2} \mathrm{~L}$ Holmes, ${ }^{2} \mathrm{R}$ Daly, ${ }^{2} \mathrm{D}$ Ryan, ${ }^{1} \mathrm{RM}$ Niven. ${ }^{1}$ University of Manchester, Manchester, UK; ${ }^{2}$ University Hospital of South Manchester, Manchester, UK

\subsection{6/thoraxjnl-2016-209333.383}

Background Omalizumab is an anti-IgE monoclonal antibody therapy used in patients with inadequately controlled persistent allergic IgE mediated asthma who require continuous or frequent treatment with oral corticosteroids. Previous studies have tried to predict a patient's response to omalizumab based on pre-treatment baseline characteristics. Most recent data has suggested that baseline blood eosinophils, serum periostin or FeNO may be predictive of response to omalizumab in the $\mathrm{T}_{\mathrm{H}} 2$ phenotype.

Aims This study will attempt to identify a characteristic that may explain why some patients suffering with severe asthma in a single severe asthma centre do not achieve a response when treated with the anti-IgE monoclonal antibody, omalizumab.

Methods The target population was represented by all patients previously treated or undergoing treatment with omalizumab at the Severe Asthma Service at University Hospital South Manchester $(\mathrm{n}=185)$. The study population was those for whom records could be found within the study period $(n=154)$. Demographic and clinical data was collected retrospectively from patient medical records.

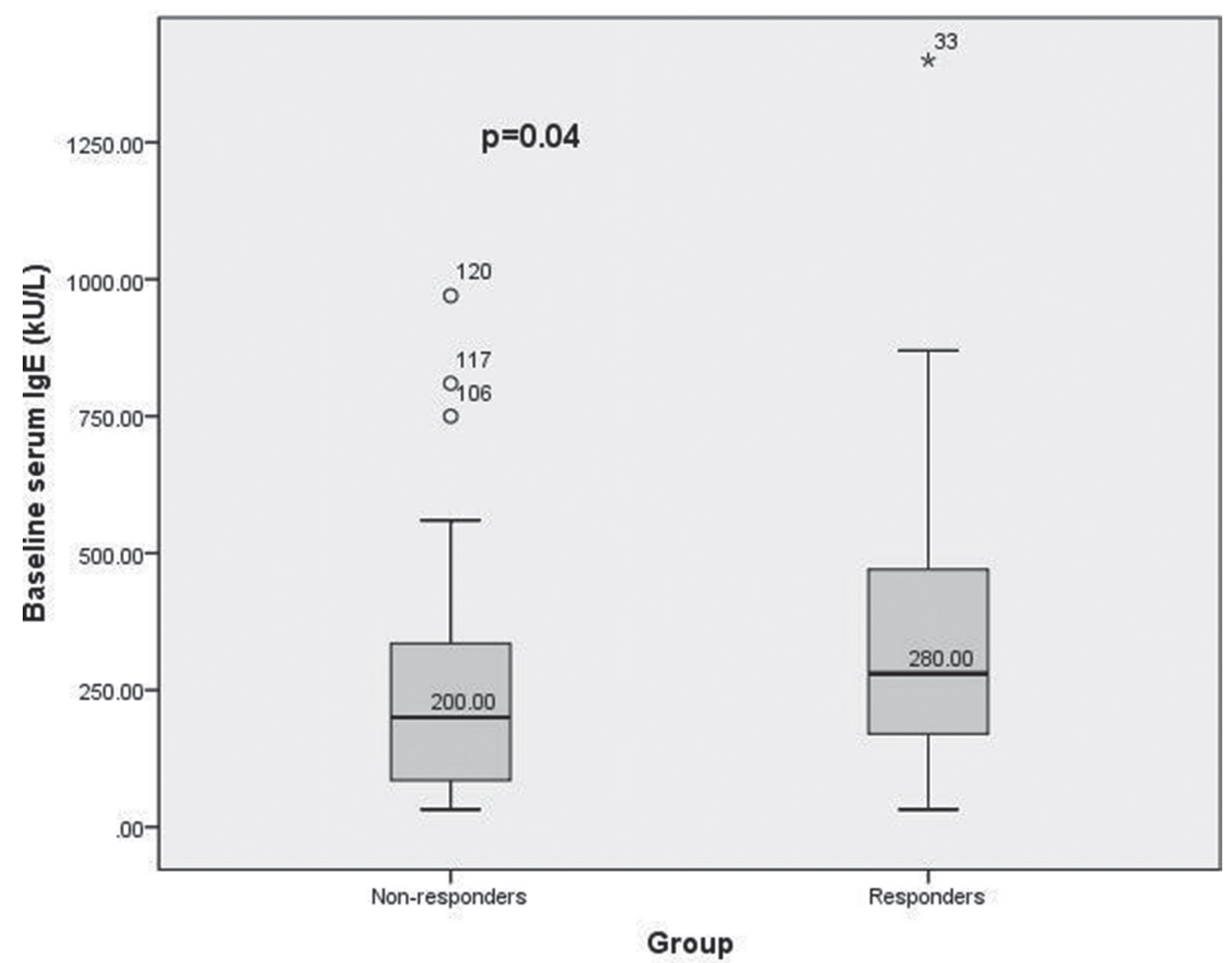

Abstract P240 Figure 1 Comparison of baseline $\lg E$ in true nonresponders and true responders 